\title{
О токовой зависимости эффективности инжекции и относительном вкладе скорости эмиссии и внутренних оптических потерь в насыщение ватт-амперной характеристики мощных импульсных лазеров $(\lambda=1.06$ мкм $)$
}

\author{
(C) А.В. Рожков \\ Физико-технический институт им. А.Ф. Иоффре Российской академии наук, \\ 194021 Санкт-Петербург, Россия \\ E-mail: rozh@hv.ioffe.rssi.ru
}

Поступила в Редакцию 5 марта 2020 г.

В окончательной редакции 23 марта 2020 г.

Принята к публикации 23 марта 2020 г.

\begin{abstract}
Приведены результаты численного моделирования токовой зависимости эффективности инжекции в активную область лазера на основе двойных гетероструктур раздельного ограничения. Показана особенность переноса носителей заряда через изотипные $N-n$-гетеропереходы на границе волноводных и активной областей. С использованием классических зависимостей теории Друде-Лоренца проведена оценка сечений рассеяния электронов $\left(\sigma_{e}\right)$ и дырок $\left(\sigma_{p}\right)$ для GaAs-волновода. Полученные значения $\sigma_{e}=1.05 \cdot 10^{-18} \mathrm{~cm}^{2}$, $\sigma_{p}=1.55 \cdot 10^{-19} \mathrm{~cm}^{2}$ и токовые зависимости эффективности инжекции позволили определить ключевую причину ограничения импульсной мощности полупроводниковых лазеров. Установлено, что внутренние оптические потери составляют незначительную долю потерь, а определяющий вклад в насыщение ваттамперной характеристики дает эмиссия дырок в волновод.
\end{abstract}

Ключевые слова: термоэлектронная эмиссия, полупроводниковый лазер, изотипный гетеропереход, насыщение ватт-амперной характеристики.

DOI: 10.21883/FTP.2020.08.49642.9389

\section{1. Введение}

Изучение многочисленных публикаций, посвященных исследованию проблемы насыщения импульсной мощности лазеров на основе двойных гетероструктур раздельного ограничения (ДГС РО) указывает на необходимость уточнения некоторых ключевых параметров и проведения дополнительного численного анализа для установления роли внутренних оптических потерь и скорости эмиссии носителей из квантово-размерной активной области.

За последние годы при исследовании проблемы насыщения токовой эффективности мощных полупроводниковых лазеров предложено и изучено несколько моделей, учитывающих различные механизмы, влияющие на оптические потери мощности в резонаторе лазерной структуры. Сечения рассеяния подвижных носителей (электронов, $\sigma_{e}$, и дырок, $\sigma_{p}$ ) являются ключевыми параметрами каждой из предложенных моделей. К сожалению, прямые экспериментальные методы непосредственного измерения влияния свободных носителей заряда сложны, а расчетные соотношения, как правило, отсутствуют и не настолько прозрачны. Поэтому определение относительного вклада внутренних оптических потерь не представляется возможным в связи с существенным различием указанных в публикациях значений $\sigma_{e}$ и $\sigma_{p}$. В качестве примера из многочисленного списка публикаций следует упомянуть, с указанием рассматриваемых механизмов и используемых значений $\sigma_{e}$ и $\sigma_{p}$, следующие работы: [1] - поглощение на свободных носителях (free carrier absorption, FCA), $\sigma_{e}=3 \cdot 10^{-18} \mathrm{~cm}^{2}$ и $\sigma_{p}=7 \cdot 10^{-18} \mathrm{~cm}^{2}$; $[2,3]$ - поглощение на свободных носителях с учетом продольной пространственной неоднородности распределения концентраций и коэффициента материального усиления $G$ в резонаторе (longitudinal spatial hole burning, LSHB), $\sigma_{e}=4 \cdot 10^{-18} \mathrm{~cm}^{2}$ и $\sigma_{p}=12 \cdot 10^{-18} \mathrm{~cm}^{2}$, $\sigma_{e}=4 \cdot 10^{-30} \mathrm{~cm}^{2}$ и $\sigma_{p}=12 \cdot 10^{-30} \mathrm{~cm}^{2} ;[4,5]-$ поглощение на свободных носителях с учетом двухфотонного поглощения (two-photon absorption, ТРА) с последующим перераспределением концентрации свободных носителей (secondary FCA effect, TPA-generated carriers, FCA TPA), $\sigma_{e}=3 \cdot 10^{-18} \mathrm{~cm}^{2}$ и $\sigma_{p}=1 \cdot 10^{-17} \mathrm{~cm}^{2}$, $\sigma_{e}=5 \cdot 10^{-19} \mathrm{~cm}^{2}$ и $\sigma_{p}=4 \cdot 10^{-17} \mathrm{~cm}^{2}$. Тем не менее авторы работ [6,7], проведя анализ результатов одномерного и двумерного моделирования для значений $\sigma_{e}=4 \cdot 10^{-18} \mathrm{~cm}^{2}, \sigma_{p}=12 \cdot 10^{-18} \mathrm{~cm}^{2}$ установили относительный вклад двух основных механизмов - поглощения на свободных носителях (FCA) и спонтанной рекомбинации в волноводных областях за счет утечки носителей из квантовой ямы (КЯ) (carrier leakage effect). Неопределенность в используемых значениях $\sigma_{e}$, $\sigma_{p}$ присутствует и в других публикациях, посвященных исследованию эффективности мощных импульсных лазеров.

Существенно меньший интерес к исследованиям эффективности инжекции в КЯ лазерных структур объясняется достаточно хорошо разработанной теорией, 
изложенной, например, в работе [8]. Использование зависимости тока термоэлектронной эмиссии в соответствии с классической теорией было признано справедливым при вычислении скорости эмиссии электронов и дырок из КЯ лазерных гетероструктур. Имеется хорошее соответствие полученных результатов теоретических и экспериментальных исследований зависимости плотности пороговых токов от температуры, времени транспорта носителей заряда к КЯ, времени захвата носителей заряда в КЯ, скорости „утечки“ электронов из КЯ в волновод от плотности заряда в КЯ [9-11]. В перечисленных публикациях основное внимание уделялось исследованиям лазерной эффективности в области плотностей токов в предпороговом и близких к пороговому режимах накачки, когда эмиссия электронов оказывает определяющее влияние. Признавая бесспорную справедливость зависимости скорости и времени эмиссии электронов и дырок от величины разрывов зон проводимости, $\Delta E_{c}$, и валентнов, $\Delta E_{v}$, следует отметить отсутствие очевидных связей указанных зависимостей с высотой потенциального барьера и распределением потенциала пространственного заряда, влияющих на эффективность инжекции носителей заряда в режимах высоких и сверхвысоких плотностей тока накачки.

Целью настоящей работы являлись: изучение особенности переноса носителей заряда через границу активной и волноводных областей с учетом эффективной высоты потенциальных барьеров при захвате электронов в КЯ и эмиссии дырок и электронов из КЯ в волноводную область; определение сечений рассеяния для неравновесных носителей заряда в волноводной области; установление вклада эмиссии заряда из КЯ и внутренних оптических потерь в токовую зависимость оптической эффективности на примере лазеров с расширенным волноводом в системе твердых растворов $\mathrm{AlGaAs} / \mathrm{GaAs} / \mathrm{InGaAs}$.

\section{2. Основные положения используемой физической модели}

Для описания токовой зависимости выходной оптической мощности лазера $P_{\text {out }}$ использовалась классическая формула, учитывающая эффективность преобразования доли рекомбинирующих носителей заряда в фотоны с энергией $h v$ для токов $I$, превышающих пороговое значение $I_{\text {th }}$. В режиме стимулированного излучения эффективность преобразования тока в свет задается внешней дифференциальной квантовой эффективностью с учетом внутренних оптических потерь $\left(\alpha_{i}\right)$ и потерь на вывод излучения из лазерного полоскового резонатора $\left(\alpha_{m}\right)$, а также внутренней дифференциальной квантовой эффективностью $\left(\eta_{i}\right)$ :

$$
\begin{aligned}
P_{\mathrm{out}} & =\eta_{i}(h v / q)\left[\alpha_{m} /\left(\alpha_{m}+\alpha_{i}\right)\right]\left(I-I_{\mathrm{th}}\right) \\
& =\eta_{s}^{d} \eta_{r}^{d} \eta_{i}^{d}(h v / q)\left[\alpha_{m} /\left(\alpha_{m}+\alpha_{i}\right)\right]\left(I-I_{\mathrm{th}}\right) .
\end{aligned}
$$

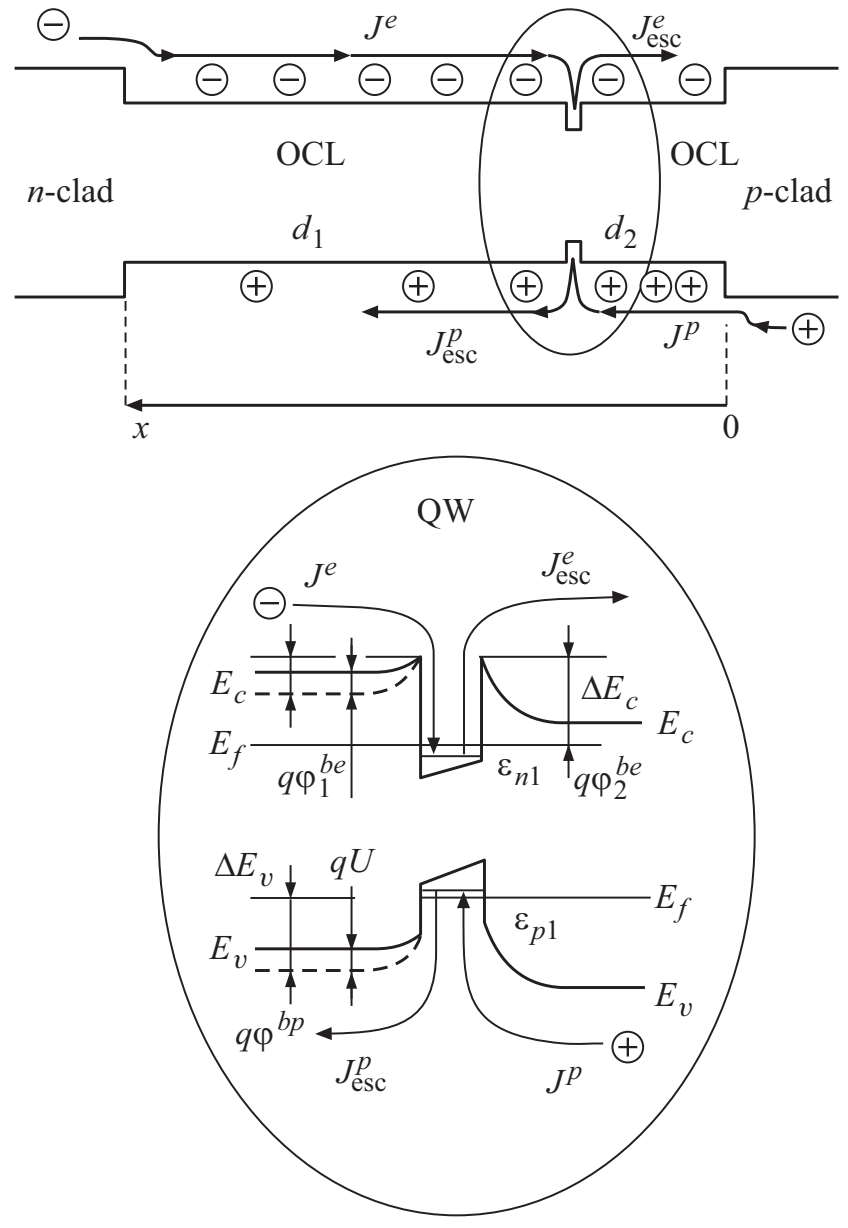

Рис. 1. Зонная энергетическая диаграмма на границах активной и волноводной областей инжекционного импульсного лазера с ассиметричным волноводом.

В формуле (1) $\eta_{i}$ представлена произведением трех составляющих, где $\eta_{s}^{d}$ - дифференциальная эффективность инжекции в пределах геометрического размера лазерного полоска, $\eta_{r}^{d}$ - дифференциальная эффективность излучательной рекомбинации и $\eta_{i}^{d}-$ дифференциальная эффективность инжекции в квантово-размерную активную область, $q$ - заряд электрона. В отсутствие токовых утечек при накачке лазерных структур и насыщении безызлучательных каналов рекомбинации $\eta_{s}^{d}, \eta_{r}^{s}$ можно с высокой точностью принять равными 1, тогда как $\eta_{i}^{d}$ имеет ярко выраженную токовую зависимость.

Для установления функциональной токовой зависимости $\eta_{i}^{d}=f(J) \quad(J-$ плотность тока $)$ исследованы особенности непрямой инжекции носителей заряда в активную область ДГС РО с учетом наличия на интерфейсах волноводных и активной областей изотипных $N-n$-гетеропереходов с соответствующими разрывами зоны проводимости, $\Delta E_{c}$, валентной зоны, $\Delta E_{v}$, распределением потенциала в области объемного заряда, определяющими высоту потенциальных барьеров $\varphi^{p p}$, $\varphi_{1}^{b e}, \varphi_{2}^{b e}$ (рис. 1). В квазистационарном случае плотность 
тока на границе КЯ и волновода со стороны $N$-эмиттера равна сумме плотностей токов дрейфа электронов, $J^{e}$, и термоэмиссии дырок, $J_{\text {esc }}^{p}$, из КЯ:

$$
J=J^{e}+J_{\mathrm{esc}}^{p}
$$

Соответственно плотность тока на границе КЯ и волновода со стороны $P$-эмиттера равна сумме плотностей диффузионного тока дырок, $J^{p}$, и термоэмиссионного тока электронов, $J_{\text {esc: }}^{e}$

$$
J=J^{p}+J_{\text {esc }}^{e}
$$

Плотность дрейфового тока со стороны $N$-эмиттера $J^{e}$ рассчитывалась в соответствии с классической термоэмиссионно-диффузионной теорией Шоттки [12], справедливой в том числе для случая изотипных гетеропереходов:

$$
\begin{aligned}
J^{e} & =q N_{c} V_{D} \exp \left(-q \varphi_{1}^{b e} / k T\right) \\
& =q N_{c} V_{D} \exp \left[-\left(\Delta E_{c}-E_{f}^{e} / k T\right)\right] \exp (q U / k T) .
\end{aligned}
$$

В (4) $V_{D}$ и $N_{c}$ - дрейфовая скорость и плотность состояний в зоне проводимости волновода, $k-$ постоянная Больцмана, $U-$ напряжение смещения на $N-n$-гетеропереходе, $q-$ заряд электрона, $T$ - температура, $E_{f}^{e}-$ положение уровня Ферми в GaAs-волноводе. В приближении Шоттки максимальное значение поля на границе волновод-КЯ достигает $(3-7) \cdot 10^{3} \mathrm{~B} / \mathrm{cm}$ в случае линейного распределения поля в области объемного заряда изотипных $N-n$-гетеропереходов при фоновом легировании в интервале $(1-3) \cdot 10^{16} \mathrm{~cm}^{-3}$. Это означает, что дрейфовая скорость $V_{D} \approx 2 \cdot 10^{7} \mathrm{~cm} / \mathrm{c}$, т.е. близка к максимальным значениям дрейфовой скорости в GaAs.

Плотности токов термоэмиссии из КЯ определялись в предположении надбарьерного переноса носителей заряда (электронов и дырок), находящихся в первой подзоне размерного квантования с энергией $\varepsilon_{e, p}^{1}=\pi^{2} \hbar^{2} /\left(2 m_{e, p}^{*} d^{2}\right)$, где $m_{e, p}^{*}-$ эффективная масса электрона или дырки в КЯ; $\hbar$ - постоянная Планка, $d$ - толщина КЯ:

$$
\begin{aligned}
J_{\mathrm{esc}}^{p}= & \left(4 \pi q m_{p}^{*} / h^{3}\right)(k T)^{2} \exp \left[-\left(q \varphi^{b p} / k T\right)\right] \\
= & \left(4 \pi q m / h^{3}\right)(k T) \exp \left[-\left(\Delta E_{c}+\Delta E_{v}-E_{f}^{p}\right) / k T\right] \\
& \times \exp (q U / k T), \\
J_{\mathrm{esc}}^{p}= & \left(4 \pi q m_{e}^{*} / h^{3}\right)(k T)^{2} \exp \left[-\left(q \varphi_{2}^{b e} / k T\right)\right] \\
= & \left.\left(4 \pi q m_{e}^{*} / h^{3}\right)(k T) \exp \left[-\left(\Delta E_{c}-E_{f}^{e}\right) / k T\right] / k T\right) .
\end{aligned}
$$

Были исследованы лазеры на основе ДГС РО с одиночной квантово-размерной активной областью из твердого раствора $\operatorname{In}_{0.3} \mathrm{Ga}_{0.7} \mathrm{As}$, имеющей толщину $d=100 \AA$. Рассчитанные значения энергии для первых уровней размерного квантования электронов и дырок составляют

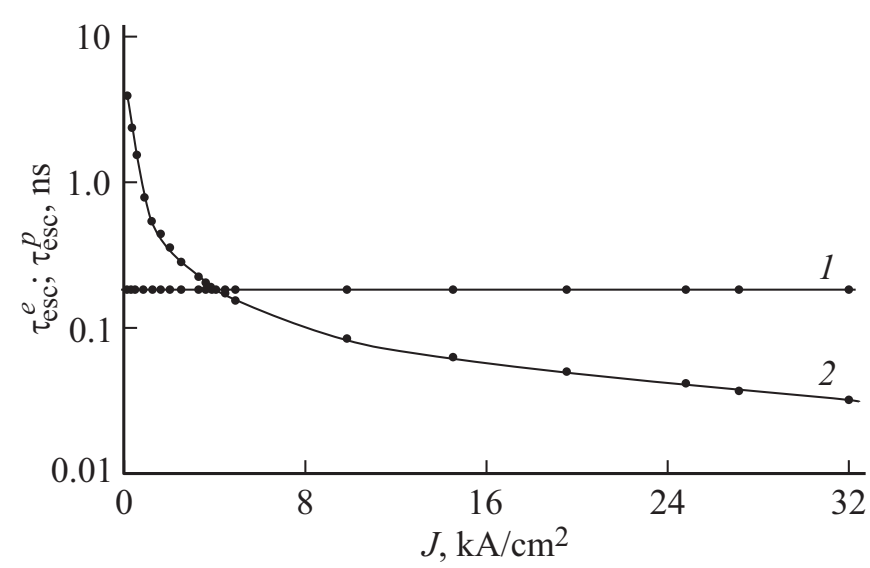

Рис. 2. Токовые зависимости времени температурной эмиссии электронов (1) и дырок (2) из квантово-размерной активной области лазера в волновод.

26 и 11 мэВ. Для материала КЯ эффективные массы дырок и электронов равны соответственно $m_{p}^{*}=0.356 m_{0}$ и $m_{e}^{*}=0.063 m_{0}$, где $m_{0}-$ масса свободного электрона. Рассчитанные эффективные плотности состояний: для двумерных (2D) электронов в зоне проводимости КЯ $-N_{c}^{2 \mathrm{D}}=6.79 \cdot 10^{11} \mathrm{~cm}^{2}$, и для 2D дырок в валентной зоне $-P_{v}^{2 \mathrm{D}}=3.84 \cdot 10^{12} \mathrm{~cm}^{2}$. Структуры содержали расширенную волноводную область на основе GaAs общей толщиной 1.5 мкм. ДГС РО имели ассиметричное расположение активной области для минимизации вклада оптических мод высшего порядка. Толщина волноводной области со стороны $N$-эмиттера составляла $d_{1}=1.2$ мкм, со стороны $P$-эмиттера $d_{2}=0.3$ мкм. При расчетах были использованы значения $\Delta E_{c}=182 \mathrm{мэВ} \mathrm{и} \Delta E_{v}=113$ мэВ. Лазер имел ширину полоска 100 мкм при длине резонатора ФабриПеро $\sim 3$ мм. Потери на вывод излучения из лазерного полоскового резонатора учитывались значением $\alpha_{m} m=5.1$.

Токовые зависимости времени температурной эмиссии из квантово-размерной активной области лазера в волновод.

В условиях квазинейтральности при равенстве концентрации неравновесных носителей в КЯ $\left(N^{\mathrm{QW}}=P^{\mathrm{QW}}\right)$ токовые зависимости времени эмиссии электронов и дырок $\tau_{\mathrm{esc}}^{e}=q d N^{\mathrm{QW}} / J_{\text {esc }}^{e}$ и $\tau_{\mathrm{esc}}^{p}=q d P^{\mathrm{QW}} / J_{\mathrm{esc}}^{p}$, имеют принципиальные различия (рис. 2). Здесь в численном моделировании использовалась линейная аппроксимация зависимости концентрации носителей в КЯ от тока накачки $N^{\mathrm{QW}}=P^{\mathrm{QW}}=J^{p} \tau_{\mathrm{ph}}$. Время жизни фотонов в резонаторе лазерной структуры $\tau_{\text {ph }}$ рассчитывалось как $\tau_{\mathrm{ph}}=1 / \alpha_{m} v_{\mathrm{gr}}=\bar{n} / \alpha_{m} c$, где $v_{\mathrm{gr}}$ и $c-$ соответственно групповая скорость фотонов и скорость света. Показатель преломления для GaAs-волновода равен $\bar{n}=3.45$. Далее будет проведена оценка внутренних оптических потерь мощности лазерного излучения $\alpha_{i}$ и в случае необходимости будут внесены поправки при численном 
моделировании токовой зависимости коэффициента инжекции в КЯ.

\section{3. Использование \\ дисперсионных соотношений теории Друде-Лоренца для оценки значений сечений рассеяния в GaAs-волноводе}

Представление вещества набором гармонических осцилляторов, используемое в теории Друде-Лоренца для описания откликов среды на воздействие электромагнитного излучения (ЭМИ), позволяет установить долю потерь мощности излучения при распространении ЭМИ в проводящей среде. Воспользуемся основными положениями дисперсионной модели для полупроводников, представленной, например, в $[13,14]$, для оценки внутренних оптических потерь в рассматриваемых лазерных структурах. При гармоническом с частотой $\omega$ поле ЭМИ, какие бы процессы ни вызывали поглощение энергии излучения, они приводят к возникновению мнимой составляющей диэлектрической проницаемости:

$$
\varepsilon^{\prime \prime}=N q^{2} / m_{e, p}^{*} \varepsilon_{0} \omega \tau /\left(1+\omega^{2} \tau^{2}\right) .
$$

Здесь $m_{e, p}^{*}$ - эффективная масса свободных носителей заряда (электронов или дырок) в GaAs-волноводе, $N$ и $q$ - концентрация и величина заряда неравновесных носителей заряда, $\tau$ - время релаксации импульса носителей заряда, которое для случая независимости от энергии имеет следующую связь с подвижностью $\mu$ и эффективной массой $m^{*}: \tau=\left(m^{*} \mu\right) / q$.

Так как распределение неравновесных носителей заряда в волноводе лазерной структуры описывается амбиполярным коэффициентом диффузии (дрейфа), то при вычислении времени релаксации импульса использовалось значение амбиполярной подвижности. Для волновода $n$-типа проводимости при типичных уровнях фоновой примеси $\sim 10^{16} \mathrm{~cm}^{-3}$ амбиполярная подвижность определялась дырочной подвижностью и с ростом тока накачки ее значение изменялось от $\mu_{p}=300 \mathrm{~cm}^{2} / \mathrm{B} \cdot \mathrm{c}$ до $2 b /(b+1) \mu_{p}=570 \mathrm{~cm}^{2} / \mathrm{B} \cdot \mathrm{c}$. Время релаксации импульса рассматривалось как мера „инерции“ единого ансамбля электрон-дырка, учитывающая отклик вещества на воздействие ЭМИ для любой координаты распределенной в волноводной области концентрации инжектированных носителей заряда $(N \sim p, n)$. В условиях квазинейтральности релаксация импульса неравновесных электронов и дырок описывалась равными временами с соответствующими значениями $\tau=\left(m_{p}^{*} \mu\right) / q$. Одновременно учитывалось различие частот продольных плазменных колебаний электронов и дырок при вычислении диэлектрической функции (7) для любых значений $N$, соответствующих плотности тока накачки лазера.

Мнимая часть комплексной диэлектрической проницаемости, вычисляемая по формуле (7), позволяет оценить значение показателя поглощения $k$ и коэффициента поглощения $\alpha$, используя классические соотношения $\varepsilon^{\prime \prime}=2 \bar{n} k$ и $\alpha=4 \pi k / \lambda$. Здесь $\bar{n}-$ показатель преломления материала волновода, $\lambda$ - длина волны излучения. Записывая функциональную зависимость коэффициента поглощения от концентрации свободных носителей заряда в виде $\alpha_{e, p}=\sigma_{e, p} N$, делаем оценку $\sigma_{e, p}-$ сечений рассеяния соответственно для электронов или дырок. Было установлено, что для мощных импульсных лазеров с $\lambda=1.06$ мкм $\left(\omega=1.78 \cdot 10^{15} \mathrm{c}^{-1}\right) \sigma_{e}=1.05 \cdot 10^{-18} \mathrm{cм}^{2}$ и $\sigma_{p}=1.55 \cdot 10^{-19} \mathrm{~cm}^{2}$. Полученные значения находятся в хорошем соответствии с данными работы [15], в которой приведены численные значения коэффициентов поглощения на свободных носителях заряда для различных полупроводников, в том числе полупроводников группы $\mathrm{A}^{\mathrm{III}} \mathrm{B}^{\mathrm{V}}$, и их зависимости от концентрации электронов. Полученные значения сечений рассеяния будут использованы для расчета токовых зависимостей коэффициента поглощения и оценки внутренних оптических потерь в волноводе при различных уровнях тока накачки.

\section{4. Определение относительного вклада скорости эмиссии и внутренних оптических потерь в насыщение ватт-амперной характеристики мощных импульсных лазеров}

При численном анализе ватт-амперных характеристик инжекционного лазера использовалась система скоростных уравнений, описывающих процессы приращения и убыли концентрации носителей заряда в КЯ. В этих уравнениях приращение концентраций задается током инжекции, а убыль - скоростью спонтанной и стимулированной рекомбинации и температурной эмиссией электронов и дырок из КЯ в волновод. Помимо полного числа носителей заряда в активной области $N^{\mathrm{QW}}\left(P^{\mathrm{QW}}\right)$ использовалось полное число фотонов в резонаторе $S$. Скорость изменения числа фотонов $d S / d t$ контролировалась увеличением их числа в результате процессов стимулированного излучения $v_{\mathrm{gr}} G S$, а также уменьшением в связи с наличием в резонаторе оптических потерь. При рассмотрении числа фотонов в резонаторе учитывались только фотоны, рожденные в результате актов стимулированного излучения, и не рассматривалось спонтанное излучение. При этом допущении $S \approx N^{\mathrm{QW}}=P^{\mathrm{QW}}$, а погрешность выполненных далее оценок не превышает значения $\tau_{\mathrm{ph}} / \tau_{d} \approx 10^{-2}$, где $\tau_{d}-$ дифференциальное время жизни носителей заряда в КЯ. Темп убыли концентрации фотонов в резонаторе задавался через время жизни фотонов $\tau_{\mathrm{ph}}$. Таким образом, система скоростных уравнений имела следующий вид:

$$
\begin{aligned}
d P^{\mathrm{QW}} / d t & =J^{p} / q-J^{p}\left(\tau_{\mathrm{ph}} / \tau_{\mathrm{esc}}^{p}\right) / q-S / \tau_{\mathrm{ph}} \\
& =J^{p} / q-J_{\mathrm{esc}}^{p} / q-S / \tau_{\mathrm{ph}},
\end{aligned}
$$




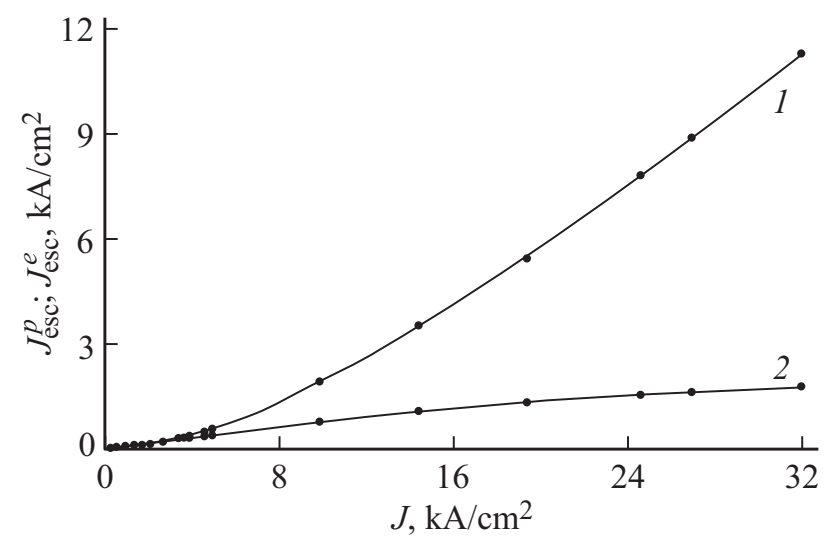

Рис. 3. Зависимости плотности токов температурной эмиссии дырок $J_{\mathrm{esc}}^{p}(1)$ и электронов $J_{\mathrm{esc}}^{e}(2)$ от плотности импульсного тока накачки $J$.



Рис. 4. Токовые зависимости относительной доли потерь на вывод излучения в сумме потерь на вывод излучения из лазерного резонатора и на поглощение на свободных носителях заряда, $\alpha_{m} /\left(\alpha_{m}+\alpha_{i}\right)(1)$, а также дифференциальной эффективности инжекции (2).

$$
\begin{aligned}
d N^{\mathrm{QW}} / d t & =J^{e} / q-J^{e}\left(\tau_{\mathrm{ph}} / \tau_{\mathrm{esc}}^{e}\right) / q-S / \tau_{\mathrm{ph}} \\
& =J^{e} / q-J_{\mathrm{esc}}^{e} / q-S / \tau_{\mathrm{ph}} .
\end{aligned}
$$

С помощью системы уравнений $(8),(9)$ для квазистационарных состояний $d P^{\mathrm{QW}} / d t=d N^{\mathrm{QW}} / d t=0$ (с учетом $P^{\mathrm{QW}}=N^{\mathrm{QW}}$ ) определялись токовые зависимости $J_{\text {esc }}^{p}$ и $J_{\text {esc }}^{e}$ (рис. 3), а также токовая зависимость дифференциальной эффективности инжекции в КЯ $\eta_{i}^{d}=f(J)=\left(J-J_{\mathrm{esc}}^{p}-J_{\mathrm{esc}}^{e}\right) / J$ (рис. 4, кривая 2). На рис. 3 приведены расчетные зависимости плотности тока термоэмиссии дырок $J_{\text {exc }}^{p}(1)$ и термоэмиссии электронов $J_{\text {esc }}^{e}(2)$ от плотности импульсного тока накачки лазера. Установлено, что в лазерах на основе ДГС РО в системе твердых растворов $\mathrm{AlGaAs} / \mathrm{GaAs} / \mathrm{InGaAs}$ с $\lambda=1.06$ мкм в диапазоне плотности токов накачки $J_{\text {th }} \leq J \leq 30 J_{\text {th }}$ ток электронной термоэмиссии превосходит ток дырочной термоэмиссии, и при $J_{\text {th }} \leq J \leq 8 J_{\text {th }}$ отношение токов термоэмиссии $J_{\mathrm{esc}}^{e} / J_{\mathrm{esc}}^{p} \geq 5$. В области высоких и сверхвысоких уровней инжекции дырочная составляю- щая тока термоэмиссии существенно превосходит электронную составляющую, и при плотности тока накачки $J=32 \kappa \mathrm{A} / \mathrm{cm}^{2}$ их отношение достигает $J_{\text {esc }}^{p} / J_{\text {esc }}^{e} \geq 6$. Приведенные на рис. 3 токовые зависимости $J_{\text {esc }}^{e}$ и $J_{\text {tsc }}^{p}$ соответствуют токовым зависимостям времени эмиссии электронов и дырок (рис. 2) и подтверждают наличие выраженной особенности заполнения КЯ неравновесными носителями заряда. Дырки в результате диффузии свободно попадают в КЯ, а для преодоления электронами потенциального барьера на границе, согласно (4), требуется фиксированный уровень положительного смещения. С ростом плотности тока уровень положительного смещения на изотипном $N-n$-переходе возрастает, что ведет к снижению потенциального барьера для дырок и росту тока температурной эмиссии дырок из КЯ в волновод в соответствии с (5). Одновременно с ростом тока растет двумерная концентрация электронов $N^{\mathrm{QW}}$ в КЯ, что приводит к смещению квазиуровня Ферми и уменьшению величины потенциального барьера $\varphi^{b e}$. В результате плотность тока температурной эмиссии электронов растет в соответствии с зависимостью (6). Выражения для плотностей токов (4)-(6) свидетельствуют о наличии как общих с классической теорией [12] закономерностей, так и характерных для двумерной системы отличий. Так, токовые компоненты $J^{e}, J_{\mathrm{esc}}^{p}$ зависящие от высоты соответствующих потенциальных барьеров $\varphi_{1}^{b e}$ и $\varphi^{b p}$, имеют выраженную вольт-амперную характеристику $J^{e} \propto \exp (q U / k T)$ и $J_{\mathrm{esc}}^{p} \propto \exp (q U / k T)$, тогда как токовая компонента $J_{\text {esc }}^{e}$ зависит лишь от степени заполнения электронами первого уровня размерного квантования активной области и определяется величиной потенциального барьера $\varphi_{2}^{b e}$ (рис. 1). Наличие экспоненциальной составляющей в вольт-амперной зависимости дырочного тока термоэмиссии объясняет суперлинейный характер его токовой зависимости от плотности тока накачки лазера.

При численном анализе ватт-амперных характеристик инжекционного лазера учитывалась токовая зависимость внутренних оптических потерь мощности лазерного излучения $\alpha_{i}=f(J)$, определяемая сечением рассеяния излучения и концентраций неравновесных носителей заряда в волноводе $\alpha_{e, p}=\sigma_{e, p} N$. Для определения распределения концентрации неравновесных носителей заряда $(N \sim p, n)$ решалась одномерная система уравнений для произвольного уровня инжекции в волноводе по обе стороны от КЯ. Запись исходной системы уравнений для токов имеет вид

$$
J=q p E \mu_{p}-q D_{p} d p / d x
$$

с граничным условием

$$
\begin{gathered}
x=0, \quad p=J L_{p} / q D_{p}, \\
J_{\mathrm{esc}}^{p}=q p E \mu_{p}-q D_{p} d p / d x
\end{gathered}
$$

с граничным условием

$$
x=0.3, \quad p=J_{\mathrm{esc}}^{p} L_{p} / q D_{p},
$$




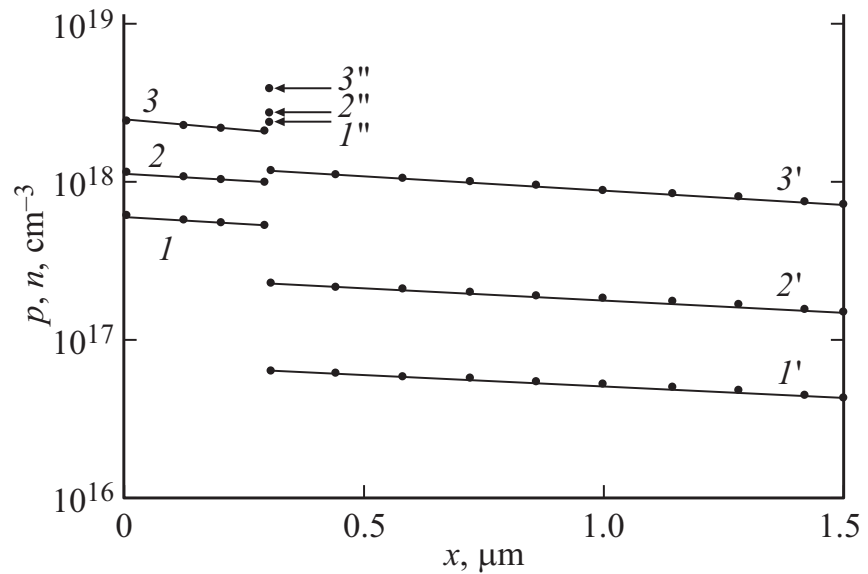

Рис. 5. Распределение концентрации неравновесных носителей заряда $(p, n)$ в волноводе со стороны $P$-эмиттера $(1,2,3)$, в волноводе со стороны $N$-эмиттера $\left(1^{\prime}, 2^{\prime}, 3^{\prime}\right)$ и в квантоворазмерной активной области $\left(1^{\prime \prime}, 2^{\prime \prime}, 3^{\prime \prime}\right)$ лазера при амплитуде импульсного тока накачки $J$, А: $1,1^{\prime}, 1^{\prime \prime}-14$, $\left(2,2^{\prime}, 2^{\prime \prime}\right)-30,\left(3,3^{\prime}, 3^{\prime \prime}\right)-100$.

$$
J^{e}=q E \mu_{e} n .
$$

Задача решалась при следующих допущениях:

- безызлучательное время жизни, подвижности основных носителей заряда (электронов) не зависят от уровня инжекции: $\tau_{e}, \mu_{e}$;

- время жизни неравновесных неосновных носителей заряда $\tau_{p}$ рассчитывалось в зависимости от уровня инжекции как $1 / \tau_{p}=1 /(A+B p)$, где $A=10^{8} \mathrm{c}^{-1}$ — коэффициент безызлучательной мономолекулярной рекомбинации через локальные центры, $B=10^{-10} \mathrm{~cm}^{3} \cdot \mathrm{c}^{-1}-$ коэффициент излучательной рекомбинации;

- электронная составляющая тока в волноводной области со стороны $N$-эмиттера обеспечивает сохранение квазинейтральности в волноводе и КЯ и соответствует скорости рекомбинации инжектированных неосновных носителей.

Исключением из уравнений (10), (11) напряженности электрического поля и с использованием взятых из работы [16] токовых зависимостей скорости дрейфа $v_{p}=\mu_{p} E$ $=(1 / q) J /\left[(b+1) p+b n^{0}\right]$ и коэффициента амбиполярной диффузии $D_{p}=\left(2 p+n^{0}\right) /\left[(b+1) p+b n^{0}\right] b D_{p}$, удалось вычислить распределения неравновесных носителей в волноводе (рис. 5). Видно, что уровень концентраций в волноводе со стороны $Р$-эмиттера существенно превосходит концентрацию фонового легирования и при плотности тока накачки $J \leq 10 \kappa \mathrm{A} / \mathrm{cm}^{2}$ достигает значений $p(n)>10^{18} \mathrm{~cm}^{-3}$. В волноводе со стороны $N$-эмиттера профиль концентрации неравновесных носителей заряда определяется величиной дырочного тока термоэмисси из КЯ. Уже при токах накачки $J \approx 5 \cdot 10^{3}$ кА/ $\mathrm{cm}^{2}$ уровень концентрации $p(n)$ в этой области волновода превышает уровень фонового легирования, что влияет на условие распространения излучения в резонаторе лазера.
С учетом ассиметричного положения КЯ в волноводе производился расчет доли внутренних оптических потерь:

$$
\begin{aligned}
\alpha_{i}= & \sum_{x=0}^{x=0.3} 0.2\left\langle\sigma_{e} n(x)+\sigma_{p} p(x)\right\rangle \\
& +\sum_{x=0.3}^{x=1.5} 0.8\left\langle\sigma_{e} n(x)+\sigma_{p} p(x)\right\rangle .
\end{aligned}
$$

Так как в лазерах с широкими волноводами фактор оптического ограничения волновода для основной моды достигает 0.99, внутренние оптические потери на основных подвижных носителях в $P$ - и $N$-эмиттерах принимались равными 0. На рис. 4 показана токовая зависимость относительной доли потерь на вывод излучения в сумме потерь на вывод излучения из лазерного резонатора и на поглощение на свободных носителях заряда $\alpha_{m} /\left(\alpha_{m}+\alpha_{i}\right)$ (1). Было установлено, что внутренние оптические потери, определяемые уровнем концентрации неравновесных носителей, инжектированных токами дырочной термоэмиссии, составляют заметную долю общих оптических потерь в волноводе. Так, для плотности тока накачки $J=32$ кА/см ${ }^{2}$ доля внутренних оптических потерь в волноводе со стороны $P$-эмиттера составила $\alpha_{i}=0.55 \mathrm{~cm}^{-1}$ и для волновода со стороны $N$-эмиттера $-\alpha_{i}=0.93 \mathrm{~cm}^{-1}$.

При вычислении ватт-амперной характеристики лазера использовалась классическая формула (1), а также рассчитанные токовые зависимости дифференциальной эффективности инжекции в КЯ $\eta_{i}^{d}$ и относительной доли потерь на вывод излучения в сумме потерь на вывод излучения из лазерного резонатора и на поглощение на свободных носителях заряда $\alpha_{m} /\left(\alpha_{m}+\alpha_{i}\right)$ (рис. 4). На рис. 6 для наглядности роли внутренних оптических

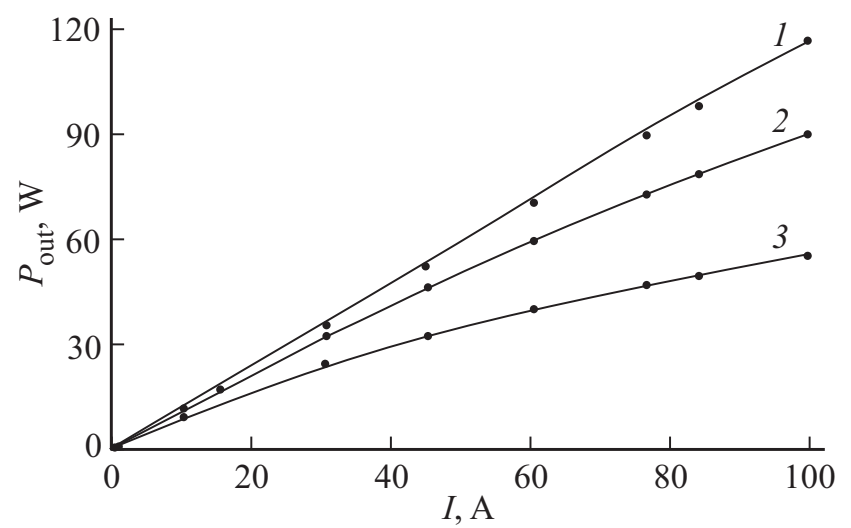

Рис. 6. Расчетные зависимости ватт-амперной характеристики лазера на основе ДГС РО в системе твердых растворов $\mathrm{AlGaAs} / \mathrm{GaAs} / \mathrm{InGaAs}: 1$ в отсутствие внутренних оптических и токовых инжекционных потерь $\left(\alpha_{i}=0\right.$ и $\left.\eta_{i}^{d}=1\right) ; 2-\mathrm{c}$ учетом внутренних оптических потерь $\left(\alpha_{i}>0\right.$ и $\left.\eta_{i}^{d}=1\right) ; 3-$ с учетом внутренних оптических и токовых инжекционных потерь $\left(\alpha_{i}>0\right.$ и $\left.\eta_{i}^{d}<1\right)$. 
и токовых инжекционных потерь приводятся кривые в отсутствие потерь (1), с учетом внутренних оптических потерь $\left(\alpha_{i}>0\right.$ и $\left.\eta_{i}^{d}=1\right)$ (2) и с учетом внутренних оптических и токовых инжекционных потерь $\left(\alpha_{i}>0\right.$ и $\left.\eta_{i}^{d}<1\right)(3)$. Кривая (3) имеет выраженный сублинейный характер, что соответствует экспериментальным ваттамперным характеристикам [1-3,6,7,17,18]. Для лазеров на основе ДГС РО в системе твердых растворов $\mathrm{AlGaAs} / \mathrm{GaAs} / \mathrm{InGaAs}$ установлено, что внутренние оптические потери составляют незначительную долю потерь, а насыщение ватт-амперной характеристики и сублинейный характер токовой зависимости коэффициента инжекции связаны с ростом скорости эмиссии дырок в волновод.

\section{5. Заключение}

Представлены результаты исследования токовой зависимости эффективности инжекции в КЯ лазеров на основе ДГС РО. Предложена физическая модель, учитывающая особенность переноса носителей заряда через границу активной и волноводных областей. Сделана оценка доли термоэмиссионных токов электронов и дырок в широком интервале плотностей токов накачки - от близких к пороговому до сверхвысоких значений $\left(>10 \kappa \mathrm{A} / \mathrm{cm}^{2}\right)$. Показано, что для плотностей токов накачки, близких к пороговым значениям, эмиссия электронов оказывает определяющее влияние на эффективность инжекции носителей заряда в КЯ, что находится в хорошем согласии с выводами авторов работ [9-11]. С ростом уровня инжекции в результате уменьшения эффективной высоты потенциального барьера существенно растет доля термоэмиссионного дырочного тока. При плотности тока накачки $J=32 \kappa \mathrm{A} / \mathrm{cm}^{2}$ плотность термоэмиссионного тока дырок достигает $J_{\mathrm{esc}}^{p} \approx 11 \kappa \mathrm{A} / \mathrm{cm}^{2}$, а плотность электронной термоэмиссии $-J_{\text {esc }}^{e} \approx 1.8 \kappa \mathrm{A} / \mathrm{cm}^{2}$.

Для оценки доли внутренних оптических потерь и анализа условий распространения лазерного излучения в волноводе использованы расчетные соотношения, связывающие электродинамические и оптические свойства лазерной гетероструктуры. Получены значения сечений рассеяния для неравновесных носителей заряда в $\mathrm{GaAs-волноводе.} \mathrm{Для} \mathrm{мощных} \mathrm{импульсных} \mathrm{лазеров} \mathrm{с}$ $\lambda=1.06$ мкм $\sigma_{e}=1.05 \cdot 10^{-18} \mathrm{~cm}^{2}, \sigma_{p}=1.55 \cdot 10^{-19} \mathrm{~cm}^{2}$. Эти значения сечений рассеяния существенно отличаются от значений, используемых, например, в $[1,17,18]$ для оценки внутренних оптических потерь, но находятся в хорошем согласии с данными работы [15].

Результаты выполненного численного моделирования и проведенный анализ позволили оценить относительный вклад двух основных механизмов - поглощения на свободных носителях и спонтанной рекомбинации в волноводных областях за счет утечки носителей из КЯ. Получено хорошее соответствие рассчитанных и наблюдаемых в эксперименте ватт-амперных характеристик $[17,18]$ для лазеров на основе ДГС РО в системе твердых растворов AlGaAs/GaAs/InGaAs. Установлено, что внутренние оптические потери составляют незначительную долю потерь, а насыщение ватт-амперной характеристики и сублинейный характер токовой зависимости коэффициента инжекции связаны с ростом скорости эмиссии дырок в волновод. Таким образом, перспектива повышения мощности импульсных полупроводниковых лазеров в значительной степени связана с возможностью подавления процесса делокализации носителей заряда из КЯ, что делает актуальным использование ассиметричных гетероструктур [19] или альтернативных вариантов - моделировано-легированных ДГС РО. Дальнейшие экспериментальные исследования должны подтвердить справедливость сделанных выводов.

\section{Благодарности}

Автор благодарит Н.С. Аверкиева и К.В. Рейха за консультации и полезные дискуссии по вопросам токовой эмиссии носителей заряда в двух- и трехмерных электронных структурах.

\section{Конфликт интересов}

Автор заявляет, что у него нет конфликта интересов.

\section{Список литературы}

[1] 3.Н. Соколова, Д.А. Веселов, Н.А. Пихтин, И.С. Тарасов, Л.В. Асрян. ФТП, 51, 998 (2017).

[2] H. Wenzel, P. Crump, A. Pietrzak, C. Roder, X. Wang, G. Erbert. Optical Quant. Electron., 41, 645 (2009).

[3] X. Wang, P. Crump, H. Wenzel, A. Liero, T. Hoffmann, A. Pietrzak, C.M. Schultz, A. Klehr, A. Ginolas, S. Einfeldt, F. Bugge, G. Erbert, G. Trankle. IEEE J. Quant. Electron., 46, 658 (2010)

[4] E.A. Avrytin, B.S. Ryvkin. Semicond. Sci. Technol., 32, 1 (2017).

[5] L.W. Hallman, B.S. Ryvkin, E.A. Avrytin, A.T. Aho, J. Viheriala, M. Guina, J.T. Kostamovaara. IEEE Photon. Tecnol. Lett., 31, 1635 (2019).

[6] J. Piprek, Z.-M. Li. IEEE Photon. Technol. Lett., 30, 963 (2018).

[7] J. Piprek. Optical Quant. Electron., 51, 60 (2019).

[8] N. Tansu, L.J. Mawst. J. Appl. Phys., 97, 054502 (2005).

[9] I.S. Shashkin, D.A. Vinokurov, A.V. Lyutetskiy, D.N. Nikolaev, N.A. Pikhtin, N.A. Rudova, Z.N. Sokolova, S.O. Slipchenko, A.L. Stankevich, V.V. Shamakhov, D.A. Veselov, K.V. Bakhvalov, I.S. Tarasov. Phys. Semicicond. Dev., 46, 1211 (2012).

[10] S.O. Slipchenko, I.S. Shashkin, L.S. Vavilova, D.A. Vinokurov, A.V. Lyutetskiy, N.A. Pikhtin, A.A. Podoskin, A.L. Stankevich, N.V. Fitisova, I.S. Tarasov. Semiconductors, 44, 661 (2010).

[11] A.E. Zhukov, N.V. Kryzhanovskaya, F.I. Zubov, Y.M. Shemyakov, M.V. Maximov, E.S. Semenova, Kr. Yyind, L.V. Asryan. Appl. Phys. Lett., 100, 021107 (2012).

[12] С. Зи. Физика полупроводниковых приборов (М., Мир, 1984) т. 1 , с. 272. 
[13] В.Б. Тимофеев. Оптическая спектроскопия объемных полупроводников и наноструктур (СПб., „Лань“, 2015) гл. 16, с. 136.

[14] Д.И. Беленко. Комплексная диэлектрическая пронищаемость. Плазменный резонанс свободных носителей заряда в полупроводниках (Саратов, Изд-во Сарат. ун-та, 1999) c. 8.

[15] H.Y. Fan. Semiconductors and Semimetals, 3, 405 (1967).

[16] Ж.И. Алферов, В.И. Корольков, И.М. Коничева, В.С. Юферев, А.А. Яковенко. ФТП, 13, 271 (1979).

[17] Д.А. Веселов, В.А. Капитонов, Н.А. Пихтин, А.В. Лютецкий, Д.Н. Николаев, С.О. Слипченко, 3.Н. Соколова, В.В. Шамахов, И.С. Шашкин, И.С. Тарасов. Квант. электрон., 44 (11), 993 (2014).

[18] Д.А. Веселов, Н.А. Пихтин, А.В. Лютецкий, Д.Н. Николаев, С.О. Слипченко, 3.Н. Соколова, В.В. Шамахов, И.С. Шашкин, И.С. Тарасов. Квант. электрон., 45 (7), 604 (2015).

[19] Ф.И. Зубов, А.Е. Жуков, Ю.М. Шерняков, М.В. Максимов, Н.В. Крыжановская, К. Yvind, Е.С. Семенова, Л.В. Асрян. Письма ЖТФ, 41 (9), 61 (2015).

Редактор Л.В. Шаронова

\section{On current dependence of the injection efficiency and the relative contribution of escape rate and internal optical losses to the saturation of power-current characteristic of pulse power lasers $(\lambda=\mathbf{1 . 0 6} \mu \mathrm{m})$}

\section{A.V. Rozhkov}

loffe Institute, 194021 St. Petersburg, Russia

\footnotetext{
Abstract The results of numerical simulation of the current dependence of the injection efficiency in the active area of the laser based on separate confinement heterostructures are presented. The feature of the transfer of charge carriers through isotype $N-n$ heterojunctions on the interface of waveguide and active areas is shown. Using the classic dependencies of the DrudeLorentz theory, the cross-section of electrons $\left(\sigma_{e}\right)$ and holes $\left(\sigma_{p}\right)$ for the GaAs waveguide was evaluated. The resulting values of $\sigma_{e}=1.05 \cdot 10^{-18} \mathrm{~cm}^{2}$ and $\sigma_{p}=1.55 \cdot 10^{-19} \mathrm{~cm}^{2}$ and current dependencies of the injection efficiency allowed to determine the root-cause reason for the pulse power saturation of semiconductor lasers. It has been established that saturation of power-current characteristics is dominated by holes escape from the active region to the waveguide and internal optical losses are lower confinement factors.
} 